\title{
Determining the effectiveness of High Resolution Melting analysis for SNP genotyping and mutation scanning at the TP53 locus Sonia Garritano ${ }^{12}$, Federica Gemignani ${ }^{1}$, Catherine Voegele ${ }^{2}$, Tú Nguyen- Dumont $^{2}$, Florence Le Calvez-Kelm² ${ }^{2}$ Deepika De Silva ${ }^{3}$, Fabienne Lesueur ${ }^{2}$, Stefano Landi ${ }^{1}$ and Sean V Tavtigian*2
}

Address: ${ }^{1}$ Department of Biology - Genetics via Derna 1, 56126 University of Pisa, Pisa, Italy, ${ }^{2}$ Genetic Susceptibility group, International Agency for Research on Cancer, Lyon, France and ${ }^{3}$ Idaho Technology Inc, Salt Lake City, Utah, USA

Email: Sonia Garritano - garritanos@iarc.fr; Federica Gemignani - fgemignani@biologia.unipi.it; Catherine Voegele - voegele@iarc.fr; Tú Nguyen-Dumont - nguyent@students.iarc.fr; Florence Le Calvez-Kelm - lecalvez@iarc.fr; Deepika De Silva - Deepika@idahotech.com; Fabienne Lesueur - lesueurf@iarc.fr; Stefano Landi - slandi@biologia.unipi.it; Sean V Tavtigian* - tavtigian@iarc.fr

* Corresponding author

Published: 17 February 2009

BMC Genetics 2009, 10:5 doi:10.1186/147|-2156-10-5

This article is available from: http://www.biomedcentral.com/I47/-2/56/10/5

(c) 2009 Garritano et al; licensee BioMed Central Ltd.

This is an Open Access article distributed under the terms of the Creative Commons Attribution License (http://creativecommons.org/licenses/by/2.0), which permits unrestricted use, distribution, and reproduction in any medium, provided the original work is properly cited.
Received: 8 September 2008

Accepted: 17 February 2009

\begin{abstract}
Background: Together single nucleotide substitutions and small insertion/deletion variants are the most common form of sequence variation in the human gene pool.

High-resolution SNP profile and/or haplotype analyses enable the identification of modest-risk susceptibility genes to common diseases, genes that may modulate responses to pharmaceutical agents, and SNPs that can affect either their expression or function. In addition, sensitive techniques for germline or somatic mutation detection are important tools for characterizing sequence variations in genes responsible for tumor predisposition. Costeffective methods are highly desirable. Many of the recently developed high-throughput technologies are geared toward industrial scale genetic studies and arguably do not provide useful solutions for small laboratory investigator-initiated projects. Recently, the use of new fluorescent dyes allowed the high-resolution analysis of DNA melting curves (HRM).

Results: Here, we compared the capacity of HRM, applicable to both genotyping and mutation scanning, to detect genetic variations in the tumor suppressor gene TP53 with that of mutation screening by full resequencing. We also assessed the performance of a variety of available HRM-based genotyping assays by genotyping 30 TP53 SNPs. We describe a series of solutions to handle the difficulties that may arise in large-scale application of HRM to mutation screening and genotyping at the TP53 locus. In particular, we developed specific HRM assays that render possible genotyping of 2 or more, sometimes closely spaced, polymorphisms within the same amplicon. We also show that simultaneous genotyping of 2 SNPs from 2 different amplicons using a multiplex PCR reaction is feasible; the data can be analyzed in a single HRM run, potentially improving the efficiency of HRM genotyping workflows.

Conclusion: The HRM technique showed high sensitivity and specificity ( 1.0 , and 0.8 , respectively, for amplicons of $<400 \mathrm{bp}$ ) for mutation screening and provided useful genotyping assays as assessed by comparing the results with those obtained with Sanger sequencing. Thus, HRM is particularly suitable for either performing mutation scanning of a large number of samples, even in the situation where the amplicon(s) of interest harbor a common variant that may disturb the analysis, or in a context where gathering common SNP genotypes is of interest.
\end{abstract}




\section{Background}

Together Single Nucleotide Polymorphisms (SNPs), rare single nucleotide substitutions, and small insertion/deletion mutations constitute the most common forms of sequence variation in the human genome. For example, Nickerson et al. [1] have estimated that the density of common SNPs (with a frequency greater than $1 \%$ ) is about 1 per $300 \mathrm{bp}$ in the overall human gene pool. Furthermore, deep resequencing studies have demonstrated that the number of rare single nucleotide substitutions and small insertion/deletion variants vastly outnumber common SNPs $[2,3]$.

During the last decade, SNPs have essentially replaced microsatellites for linkage and/or association studies [4,5] and genome-wide association studies with phase 2 and phase 3 confirmations have now provided overwhelming evidence of association on common SNPs with a number of diseases [6,7]. SNPs are also becoming of interest in pharmacogenetics, because some of them are associated with significant differences in biological response to pharmaceutical agents $[8,9]$.

Heavy interest in SNPs has led to the development of different genotyping methods: some of them are targeted to the analysis of one or few SNPs $[10,11]$, and others are designed to scan the whole genome $[12,13]$. Modern genotyping equipment has driven the per genotype cost for very large-scale SNP genotyping studies quite low. In addition, clonal sequencing technologies may drive the cost of moderate sensitivity resequencing studies very low $[14,15]$. However, these technologies are actually geared to what are essentially industrial scale genetic studies and arguably to not provide useful solutions for small laboratory investigator-initiated projects.

Interest in fast and reliable methods of mutation screening is increasing as well. Such methods are desirable for case-control mutation screening studies and highthroughput somatic (tumor) mutation screening studies $[16,17]$, aiding the identification of new genes involved in carcinogenesis. They are also desirable for detecting genes responsible for drug-resistance in micro-organisms [18], and for detecting genes that modify growth, resistance to parasites, or yield in plants [19].

Many techniques have been developed to discover genomic variation, including those based on HPLC (High Performance Liquid Chromatography), electrophoretic conformational changes, and enzymatic or chemical cleavage reactions [20]. The goal of these screening techniques is to reduce the use of DNA sequencing and control costs while maintaining sensitivity and specificity. The HRM technique has been used to mutation scan the coding sequences of several clinically important genes
[21-26]. For instance, 3 studies have reported mutation screening of TP53 exonic regions $[21,22,27]$. In this manuscript, we describe lessons learned from a larger scale application of HRM to mutation screening and genotyping at the entire TP53 locus. First, we assayed (in terms of sensitivity and specificity) the HRM technique, by comparing the results with the classic Sanger sequencing method, used here as the gold standard reference. Second, we propose solutions for genotyping challenges (discrimination of the 3 genotypes, simultaneous genotyping of 2 or more SNPs) that are sometimes encountered when using a classical HRM approach.

\section{Methods}

\section{Origin of DNA samples}

Mutation screening of the entire TP53 locus was performed on 47 DNA samples including lymphocyte DNA from 25 Li-Fraumeni patients, DNA from lymphoblastoid cell lines derived from 15 familial breast cancer patients, and DNA from 7 hemizygous (at the TP53 locus) breast tumor cell lines (Garritano et al, in preparation).

Genotyping of 30 SNPs located within the TP53 locus was performed on 270 DNA samples from the Coriell Repository, corresponding to 90 Caucasians, 90 East Asians, and 90 Africans.

This mutation screening and genotyping project received approval from the IARC Institutional Review Board and from the Brazilian center from which we received the $\mathrm{Li}$ Fraumeni patient samples. It was conducted according to the Declaration of Helsinki Principles.

\section{Mutation screening/SNP discovery using HRM}

PCR was performed in $8 \mu \mathrm{l}$ reactions containing $20 \mathrm{ng}$ of template DNA, $1.5 \mathrm{mM} \mathrm{MgCl}{ }_{2}, 265 \mu \mathrm{M}$ dNTP, $400 \mathrm{nM}$ forward and reverse primers, 0.8X LCGreen ${ }^{\circledast}$ Plus (Idaho Technology, Salt Lake City, Utah, USA), $0.04 \mathrm{U} / \mu \mathrm{l}$ of Platinum $^{\circledast}$ Taq Polymerase, and $1 \times$ PCR buffer supplied by the manufacturer (Invitrogen, Paisley, Scotland).

The HRM process consists in performing the PCR in the presence of the DNA binding dye LC Green ${ }^{\circledast}$, monitoring the progressive change in fluorescence caused by release of the dye from a DNA duplex as it is denatured by increasing the temperature, collecting a high resolution melting curve, and identifying the samples with melting curve aberrations indicative of the presence of a sequence variant. Fluorescence intensity as a function of temperature, monitored by the LightScanner ${ }^{\circledast}$ instrument (Idaho Technology, Salt Lake City, Utah, USA), can reveal very small changes in the melting curve shape, when analyzed with the LightScanner ${ }^{\circledast}$ software using the "Scanning" mode (Idaho Technology, Salt Lake City, Utah, USA). 


\section{Genotyping using HRM}

We designed pairs of primers flanking each SNP [See Additional file 1] to amplify DNA fragments shorter than $400 \mathrm{bp}$. In some instances, HRM can directly discriminate all 3 genotypes (common homozygotes, heterozygotes and rare homozygotes) of a polymorphism. However, for the majority of TP53 amplicons, genotyping using spikein control DNA was performed to allow distinction of rare homozygotes from common homozygotes. In brief, genomic DNAs were mixed with an equal amount of DNA from a known major allele homozygous subject to allow formation of heteroduplexes. This strategy converts the minor allele homozygotes into heterozygotes, rendering them distinguishable from the major allele homozygous samples. The scoring of genotypes obtained with spike-in experiments was managed via automated procedures. For instance, we have developed a Laboratory Information Management Systems (LIMS) where results generated from a standard HRM genotyping plate and a corresponding spike-in genotyping plate are automatically converted into a final genotype call [28]. The program is also capable of rejecting samples that show unacceptable calls.

For amplicons containing two or more SNPs, sensitivity of mutation scanning may be decreased by producing complex melting curve data, and a different genotyping strategy had to be applied. This second strategy relies on an unlabelled probe-based genotyping analysis followed by mutation scanning, where the probe is designed to target the SNP(s) of interest. Both probe-amplicon duplex and whole amplicon duplex melting regions can be observed from the same melting run, in two distinct temperature windows, allowing genotyping and mutation scanning analyses to be performed simultaneously. Stratification of the samples according to their genotypes at the common variant positions prior to mutation scanning analysis reduces the noise and enhances the sensitivity for the detection of rare or unknown variants.

In practice, unlabeled 3' blocked probes targeting each common SNP were designed. PCR were performed in presence of a DNA dye (Here LC Green ${ }^{\circledR}$ ) and oligonucleotides serving as probes were blocked at the 3 ' end to prevent extension during amplification. All genotyping assays were performed as a nested PCR, to ensure a good amplification of the region of interest. The primary PCR used standard conditions, whereas the secondary PCR included the unlabelled probe $(500 \mathrm{nM})$ and was asymmetric so that more copies of the strand to which the probe anneals were produced. The ratio between the nested PCR primers was 1:5 (100 nM:500 nM) with an excess of the primer for the strand that is complementary to the probe. This favours probe-target annealing and reduces competition with the complementary strand [29]. Thus, this protocol produced sufficient double-stranded product for amplicon melting and enough single stranded product for probe annealing [30]. The analysis proceeds in two steps. The first step consists in analyzing the melting curve in the region corresponding to the probe $T_{m}$. This step stratifies the samples into three groups based on the genotypes of the common SNP. The second analysis step consists in performing mutation scanning of the genotype-defined subgroups in the region corresponding to the amplicon $\mathrm{T}_{\mathrm{m}}$, to identify the samples that are heterozygous for any rare sequence variants. For the amplicon containing SNPs rs9894946 (common) and rs17883532 (rare) the probe was designed to perfectly complement the rs9894946 T allele (GGAGCTCAGTACTGCCTGCCC, the variable nucleotide is indicated in bold). For the amplicon containing SNPs rs858528, rs1641548, and rs1641549, two probes were designed. The first probe was designed to perfectly complement the rs858528 $\mathrm{G}$ allele (GCAGAGCGAGACTCAAAA). The second probe was designed to complement the rs1641548 $\mathrm{G}$ allele and rs1641549 A allele (TTAACCGGGCATGATGGCAG, the variable nucleotides corresponding to SNPs rs1641548 and rs1641549 are indicated in bold). Probes were designed to have different $\mathrm{T}_{\mathrm{m}}\left(63^{\circ} \mathrm{C}\right.$ and $54^{\circ} \mathrm{C}$, respectively), in order not to interfere with each other in the melting data analysis.

\section{Results \\ Mutation Scanning}

During the course of a project to mutation screen the entire TP53 locus by direct resequencing from a set of 47 samples, we took delivery of a High Resolution Melt instrument. To assess the sensitivity and specificity of HRM for mutation scanning, we undertook mutation screening of the last 21 TP53 amplicons and of 1 amplicon corresponding to the proximal promoter region of the gene (from a total of 67 amplicons) by both full-sequence resequencing and HRM in a single pass experiment (Table 1).

Nine of the amplicons were $<400 \mathrm{bp}$ in length, with an average length of $286 \mathrm{bp}$. For these, the sensitivity and specificity of HRM for sequence variant detection were 1.0 (38 true positive/(38 true positive +0 false negative)), and 0.83, (295 true negative/(60 false positive +295 true negative)), respectively.

Thirteen of the amplicons were $>400 \mathrm{bp}$ in length, with an average length of $544 \mathrm{bp}$. For these, the sensitivity and specificity of HRM for sequence variant detection were 0.81 (105 true positive/(105 true positive +23 false negative)), and 0.84 , (339 true negative/(69 false positive + 339 true negative)), respectively. Of note, the variant rs 17551157, insertion of a cytosine following a 7 cytosine mononucleotide run in the TP53 promoter region, was undetectable in an amplicon of $653 \mathrm{bp}$. 
Table I: Oligonucleotide primer sequences used for comparison of HRM and sequencing sensitivity and specificity.

\begin{tabular}{|c|c|c|c|c|}
\hline Amplicon & Forward sequence $5^{\prime}>3^{\prime}$ & Reverse sequence $5^{\prime}>3^{\prime}$ & Location & size \\
\hline 3 & CGGGACGTGAAAGGTTAGAA & TTTTGGGGTGGAAAATTCTG & promoter & 653 \\
\hline 39 & TGGCCATCTACAAGCAGTCA & ACACGCAAATTTCCTTCCAC & exon5-intron5 & 211 \\
\hline 40 & CATGAGCGCTGCTCAGATAG & CAGTTGCAAACCAGACCTCA & exon6 & 234 \\
\hline 41 & GTGGAAGGAAATTTGCGTGT & TTGCACATCTCATGGGGTTA & intron6 & 212 \\
\hline 43 & TGGCTCTGACTGTACCACCA & TCТАСТСССАAССАСССТTG & intron 7 & 371 \\
\hline 44 & CTGGAAGACTCCAGGTCAGG & AGCTGTTCCGTCCCAGTAGA & intron7 & 383 \\
\hline 46 & GCGCACAGAGGAAGAGAATC & TGAAAGCTGGTCTGGTCCTT & intron9 & 452 \\
\hline 47 & GCAGTGATGCCTCAAAGACA & GCAGGCTAGGCTAAGCTATGA & intron9 & 280 \\
\hline 48 & TGACTTTGCCTGATACAGATGC & TAGCTACTGGGGAGGCAGAG & intron9 & 596 \\
\hline 49 & GGCCTGCCTAGCCTACTTTT & GTAGCAGGCGCTTGTAGTCC & intron9 & 578 \\
\hline $50 \mathrm{~B}$ & GACTACAAGCGCCTGCTACC & TTTCATGCAACCATGCTGTT & intron9 & 614 \\
\hline 51 & CCCTACAGTTGGGCAAAGTC & CGACTGTGCCTCGTTTCTTT & intron9 & 491 \\
\hline $52 \mathrm{~A}$ & CCTGGGCGATAGAGTGAGAC & GGCTGGACTCAAACTCTTGG & intron9 & 134 \\
\hline $52 B$ & GTCGCATGCACATGTAGTCC & CTTGAGTTCCAAGGCCTCAT & intron9 & 635 \\
\hline 53 & АСTTСTСССССТССТСТGTT & CCTGGGTTTGGATGTTCTGT & exon 10 -intron 10 & 348 \\
\hline 55 & TATACTCAGCCCTGCCATGC & GGACTTCAGGTGGCTGTAGG & intron 10 & 603 \\
\hline 57 & TTTGGGTCTTTGAACCCTTG & GTGGTTTCAAGGCCAGATGT & exon I I (3'UTR) & 400 \\
\hline 58 & GGCCCACTTCACCGTACTAA & AAGCGAGACCCAGTCTCAAA & exon II (3'UTR) & 485 \\
\hline 59 & AAGGAAATCTCACCCCATCC & AAATGCAGATGTGCTTGCAG & exon II (3'UTR) & 456 \\
\hline 60 & TTGAGACTGGGTCTCGCTTT & CAGTCTCCAGCCTTTGTTCC & & 566 \\
\hline 61 & AAAACTTTGCTGCCACCTGT & ATCCTGCCACTTTCTGATGG & & 415 \\
\hline 62 & GCCTCTCACCAAGGATTACG & СCTGGACAGTAGCACCCACT & & 535 \\
\hline
\end{tabular}

The joint dropout rate from PCR, sequencing, and or HRM was $6.8 \%$. Neither PCR-sequencing nor PCR-HRM had a single pass dropout rate exceeding $5 \%$, thus staying above our general research mutation screening success rate target of $95 \%$.

\section{Genotyping}

We performed genotyping of 30 SNPs located within the TP53 locus on 270 DNA samples from the Coriell Repository.

In some cases, it was possible to distinguish directly the three different genotypes of a SNP using standard HRM analysis of the amplicon of interest. Figure 1 displays the melting curve analysis of the SNP rs9903378, a T>G substitution. In this experiment, it was possible to discriminate the three groups corresponding to each genotype directly (common homozygotes TT, heterozygotes TG, and rare homozygotes GG) (Figure 1). This SNP resides in a T-rich sequence that has a low melting temperature. The GG samples have a melting curve different from the common homozygotes TT; evidently, the G interrupts the long poli-Ts and markedly increases the melting point of the amplicon.

However, we found direct detection of minor allele homozygotes to be the exception rather than the rule, at least when using the mutation scanning approach for amplicons in the $200 \mathrm{bp}$ to $400 \mathrm{bp}$ length range. In this context, spike-in experiments provided an approach to detection of minor allele homozygotes. As an example, melting analyses for the SNP rs17881035 are displayed in Figure 2 (panels A, B). Applying standard melting curve analysis (Figure 2A), we observed that the heterozygous AG samples have a distinct melting curve profile compared to the common homozygote AA samples. However, the minor allele homozygote GG samples were not distinguished from the common homozygous AA samples because the difference between their $\mathrm{T}_{\mathrm{m}}$ was insufficient. In a second experiment (Figure 2B), each sample was mixed with an equal quantity of DNA from an AA homozygote (a pre-PCR spike-in experiment). This strategy in effect converts the minor allele GG homozygotes into GT heterozygotes, rendering them distinguishable from AA samples. In some instances, HRM can directly discriminate all of the genotypes of an amplicon that contains two SNPs. As an example, a 130 bp amplicon carrying SNPs rs17880560 and rs1614984 is displayed on Figure 3.

Nevertheless, we have encountered examples where HRM cannot discriminate heterozygous samples for SNP1 from heterozygous samples for SNP2. In Figure 4 (panels A, B, $\mathrm{C})$, we present an example and solution for an amplicon that contains two SNPs (rs9894946 and rs17883532) not directly distinguishable from each other. Heterozygous samples for either the first or the second SNP show almost indistinguishable melting curves (Figure 4A). We then 


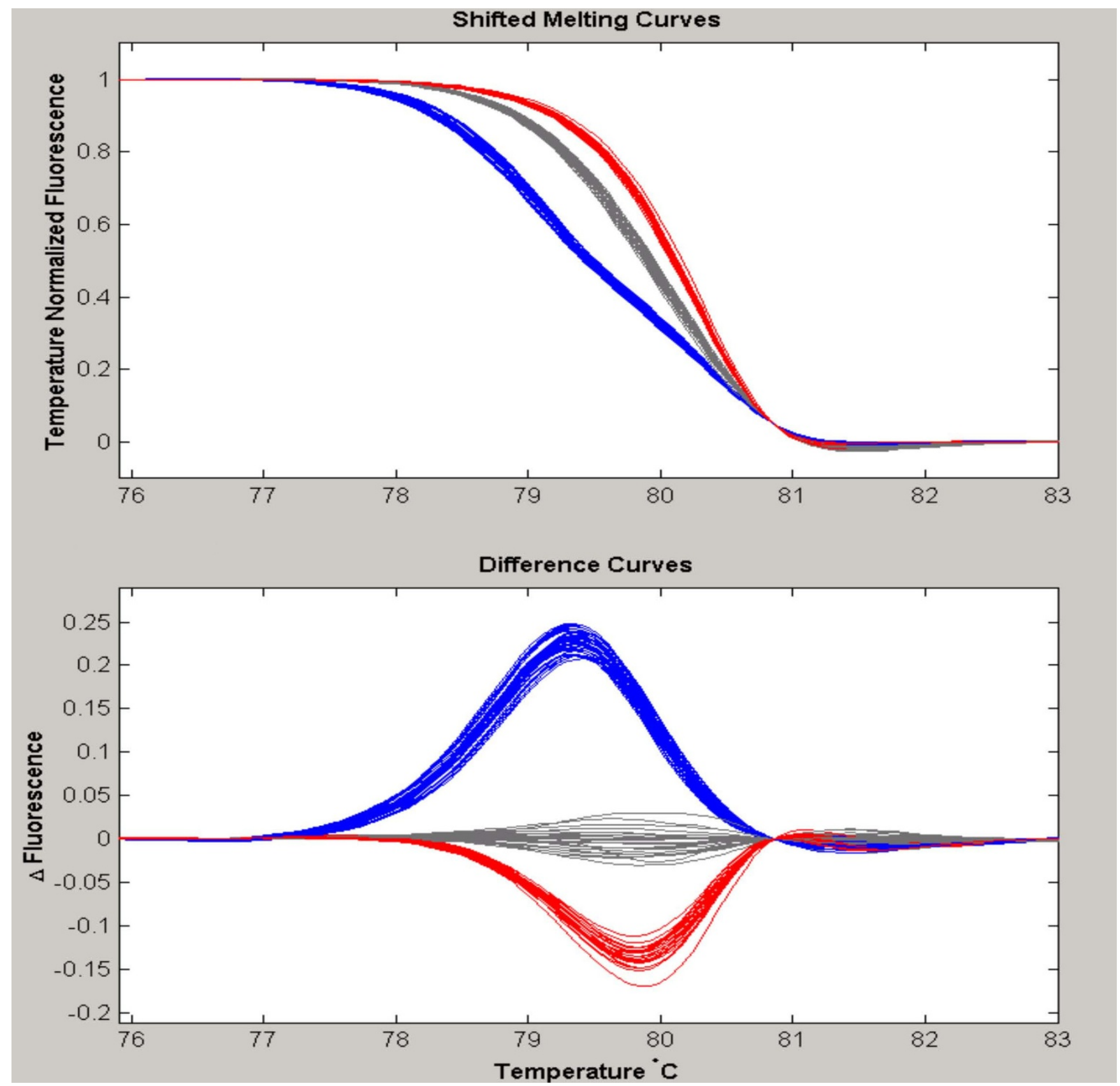

\section{Figure I}

Genotyping of SNP rs9903378. The three groups are well distinguished: TT in grey, GG in red and TG in blue.

designed an unlabeled 3' blocked probe that hybridizes to the region of sequence specific for the more common SNP rs9894946 [31]. Results are displayed in Figure 4 panels B, C. The first analysis step stratifies the samples into three groups based on the genotypes of rs9894946 (Figure 4B). In a second analysis step, mutation scanning of the genotype-defined subgroups in the region corresponding to the amplicon $\mathrm{T}_{\mathrm{m}}$ is performed, in order to identify the samples that are heterozygous for any other rare sequence variants. In this example, we found three heterozygous subjects for the SNP rs17883532 in 270 samples (Figure $4 \mathrm{C}$ ). All these heterozygous subjects were homozygous for the major allele of SNP rs9894946. This solution is acceptable only if the second SNP is rare because the mutation scanning applied after the stratification of samples according the melting profile of the common SNP targeted by the unlabeled probe may not distinguish rare from common homozygous samples. 

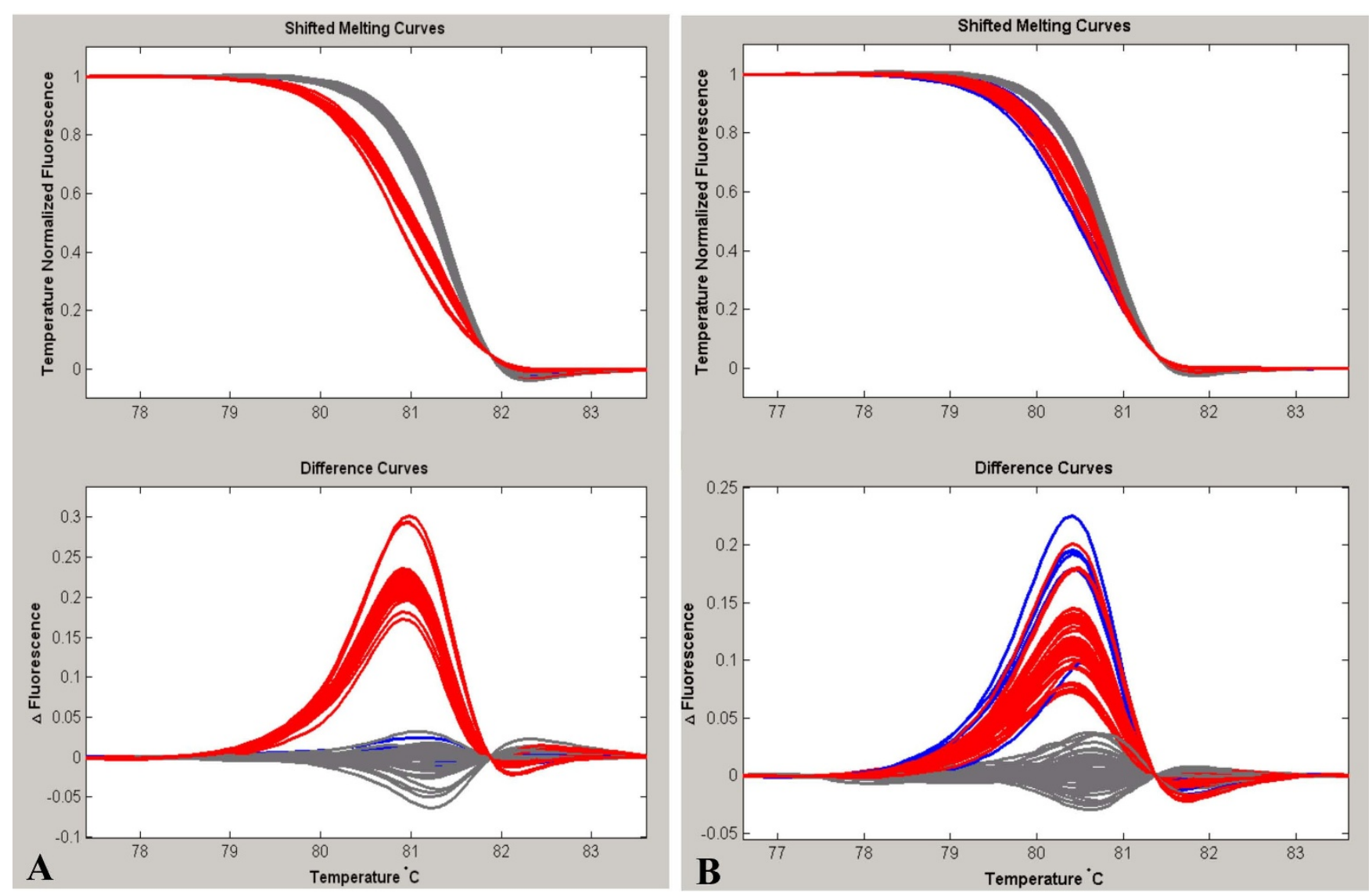

\section{Figure 2}

Genotyping using spike-in control DNA to distinguish common homozygotes from rare homozygotes. A. The melting curves of AG heterozygotes (in red) are distinguished from homozygous AA (in grey). Homozygous GG samples (in blue) are not distinguished from the common AA homozygous samples.B After spike-in of a homozygous AA sample, the GG samples are converted into AG heterozygotes (in blue), and they are now distinguished from AA samples.

During the course of our TP53 study, we faced another particular situation, where two or more common SNPs lie in the same amplicon. In such a case, it may be necessary to use more than one unlabelled probe. For instance, one of our TP53 amplicons contains two SNPs: rs1641548 and rs1641549. These are only 4 bp apart, and both are A-to$\mathrm{G}$ variants. Samples that are heterozygous for either the first or the second SNP have essentially indistinguishable melting curves (Figure 5A). Moreover, this amplicon also contains the SNP rs858528. In this case, two probes were designed. The first probe was designed to perfectly complement the rs858528 G allele. The second probe was designed to complement the rs1641548 G allele and rs1641549 A allele. Thus, homozygotes for the rs858528/ rs1641548/rs1641549 haplotype G-G-A match both probes exactly and therefore have the highest $\mathrm{T}_{\mathrm{m}}$ across the compound-melting interval. Figure $5 \mathrm{~B}$ and $5 \mathrm{C}$ show various genotypes combinations of the three SNPs found in our sample series.
Finally, we evaluated whether the different genotypes from 2 independent PCR products could be discriminated from one single melting curve analysis. Since the amplicon containing SNP rs9903378 (which can be directly genotyped, see Figure 1) and the amplicon containing SNP rs9894946 (for which a specific probe had to be designed, see Figure 4) showed different $\mathrm{T}_{\mathrm{m}}$ (range 75$80^{\circ} \mathrm{C}$ and $90-95^{\circ} \mathrm{C}$, respectively), they were selected to conduct the experiment. Both SNP containing fragments were amplified in a single PCR, and HRM analysis was conducted on the multiplex PCR product. In this last experiment, conditions of the multiplex PCR slightly differed from conditions of the simplex PCR, in order to achieve simultaneous amplification of both amplicons in a single reaction. In particular, the primer concentration for the amplicon containing the rs9903378 was decreased from $400 \mathrm{nM}$ to $300 \mathrm{nM}$ because at higher concentration only this amplicon was amplified (data not shown). Melting curves of the multiplex PCR products showed different patterns depending on the genotype combinations for the 


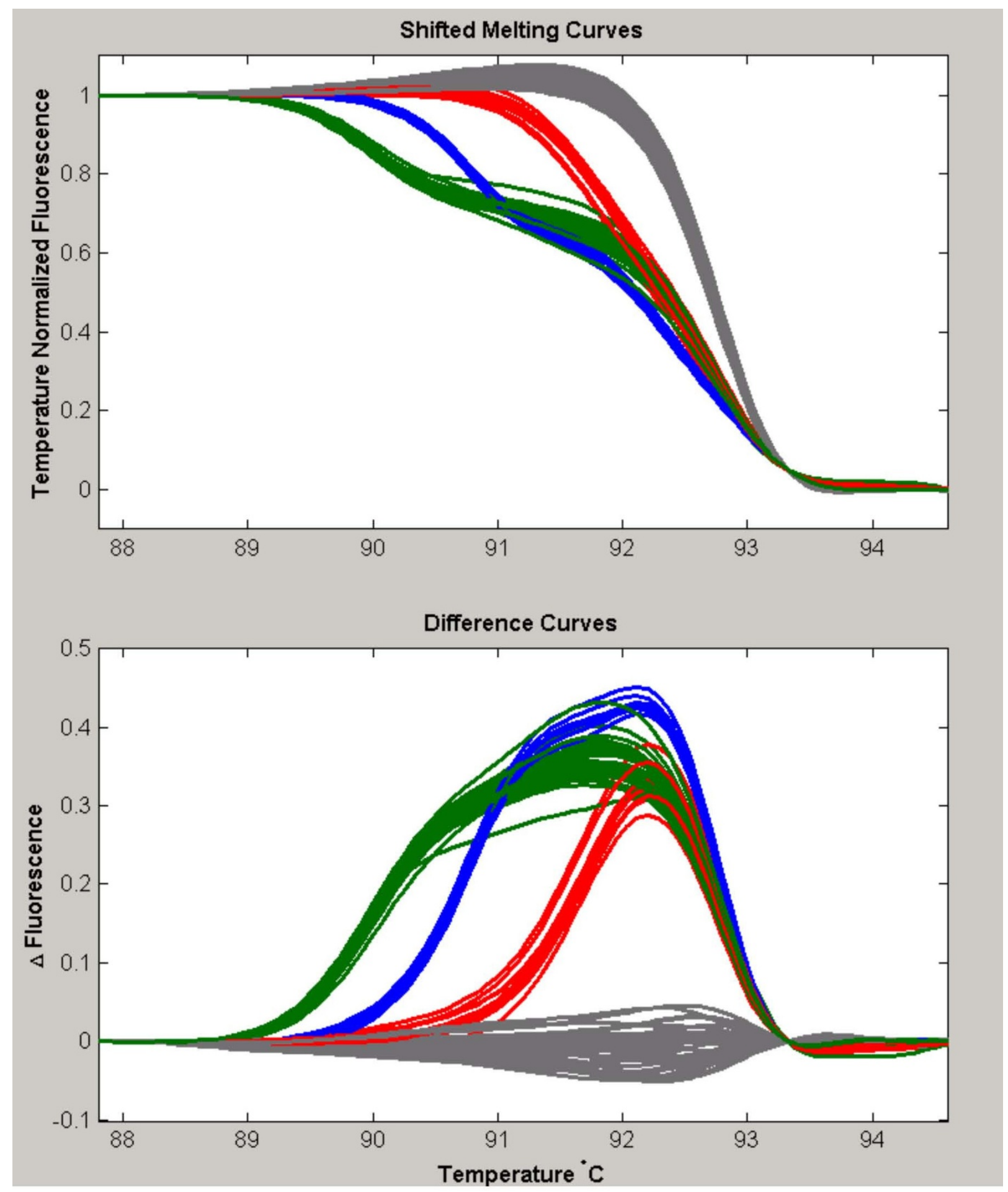

Figure 3

Simultaneous genotyping of two or more SNPs within the same amplicon using the classic HRM approach. There are two SNPs in this amplicon: rs I7880560 and rs I6I4984. In grey, samples homozygous for both SNPs; in red, samples homozygous for rs I7880560 (delCACGGC/delCACGGC) and heterozygous for rs I6I4984 (C/T). In blue, samples homozygous for rsI6I4984 (C/C) and heterozygous for rs 7880560 (insCACGGC/delCACGGC). In green, samples heterozygous for both SNPs. 


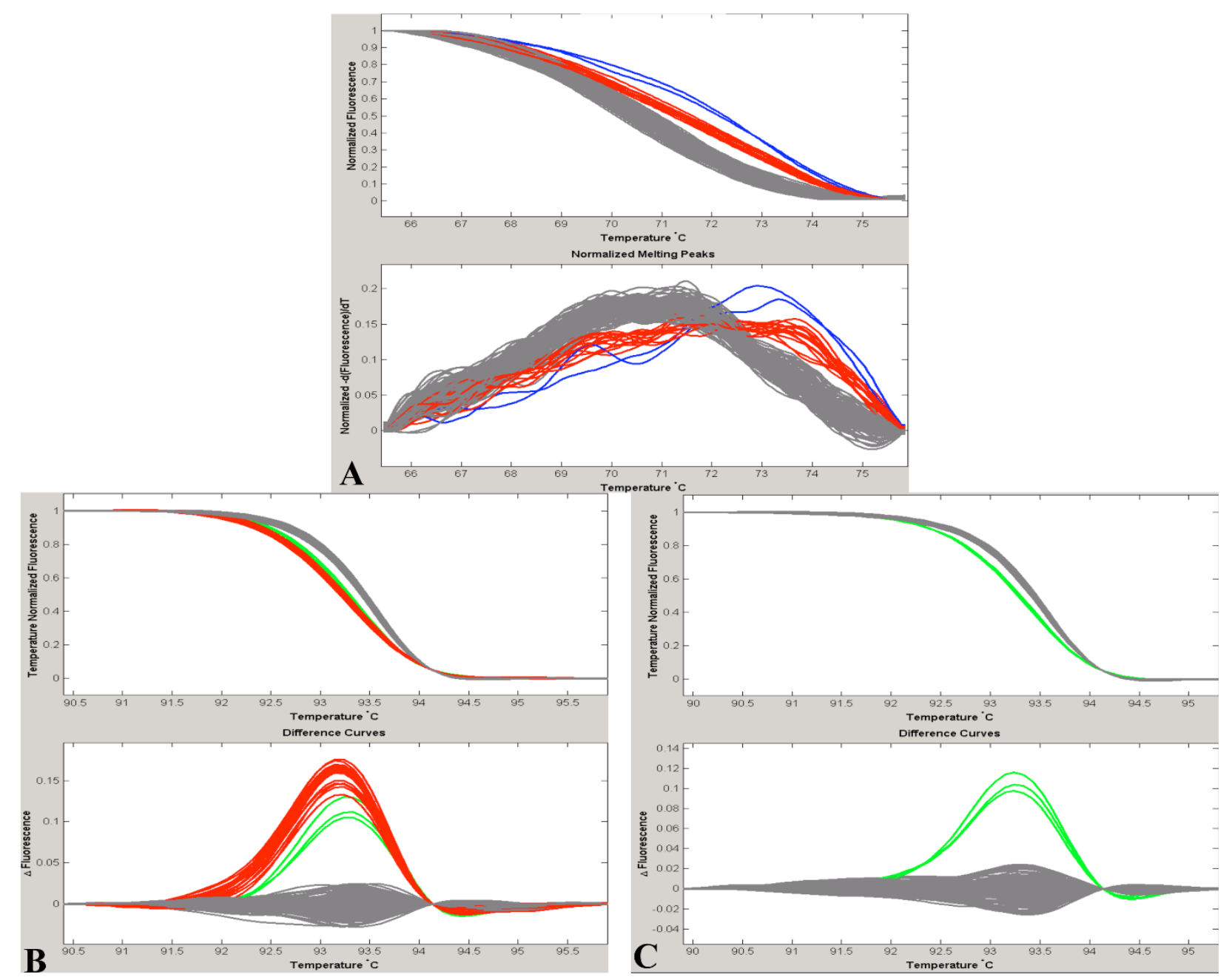

Figure 4

Simultaneous genotyping of common SNP rs9894946 and rare SNP rs 17883532 . A. In mutation scanning mode, heterozygous samples for either the first (in red) or the second SNP (in green) have virtually indistinguishable melting curves. B. In genotyping mode using an unlabeled probe for rs 9894946 , the 3 genotypes are distinguisable (CC in gray, CT in red, TT in blue). C. Mutation scanning of homozygous rs 9894946 CC subset reveals heterozygous rs I 7883532 CT (In green).

2 SNPs (Figure 6). Using the "genotyping" mode of LightScanner $^{\circledast}$ software, it was possible to distinguish them from each other in a single melting curve analysis (Figure $6 \mathrm{~A})$. However, especially when analyzing a large number of samples (>80), resolution can be improved by performing the HRM analysis of the multiplex PCR products in two steps, that is by analyzing the 2 melting regions corresponding to the 2 DNA fragments separately (Figure 6B and $6 \mathrm{C}$ ). Thus, our results demonstrate that HRM genotyping of multiplex PCR products is feasible and cost and time effective.

\section{Discussion}

In this manuscript, we assessed the sensitivity and specificity of HRM for mutation screening by comparing it head to head with the direct resequencing of 21 TP53 locus amplicons on 47 DNA samples. A second application of the HRM analysis was the genotyping of 30 known SNPs within this gene on 270 DNA samples.

In mutation scanning mode, the sensitivity and specificity of HRM were 1.0 and 0.80 , respectively, for amplicons of $<400 \mathrm{bp}$, and 0.81 and 0.84 , respectively, for amplicons of $>400 \mathrm{bp}$.

Recent studies have validated HRM for screening of number genes of clinical significance [21-26]. These studies also reported a sensitivity of HRM close to $100 \%$, except in the situation were amplicons have a high GC content [26]. We also encountered a similar situation with one TP53 GC-rich amplicon (see below). However, in the previous studies, the HRM technique was evaluated only 

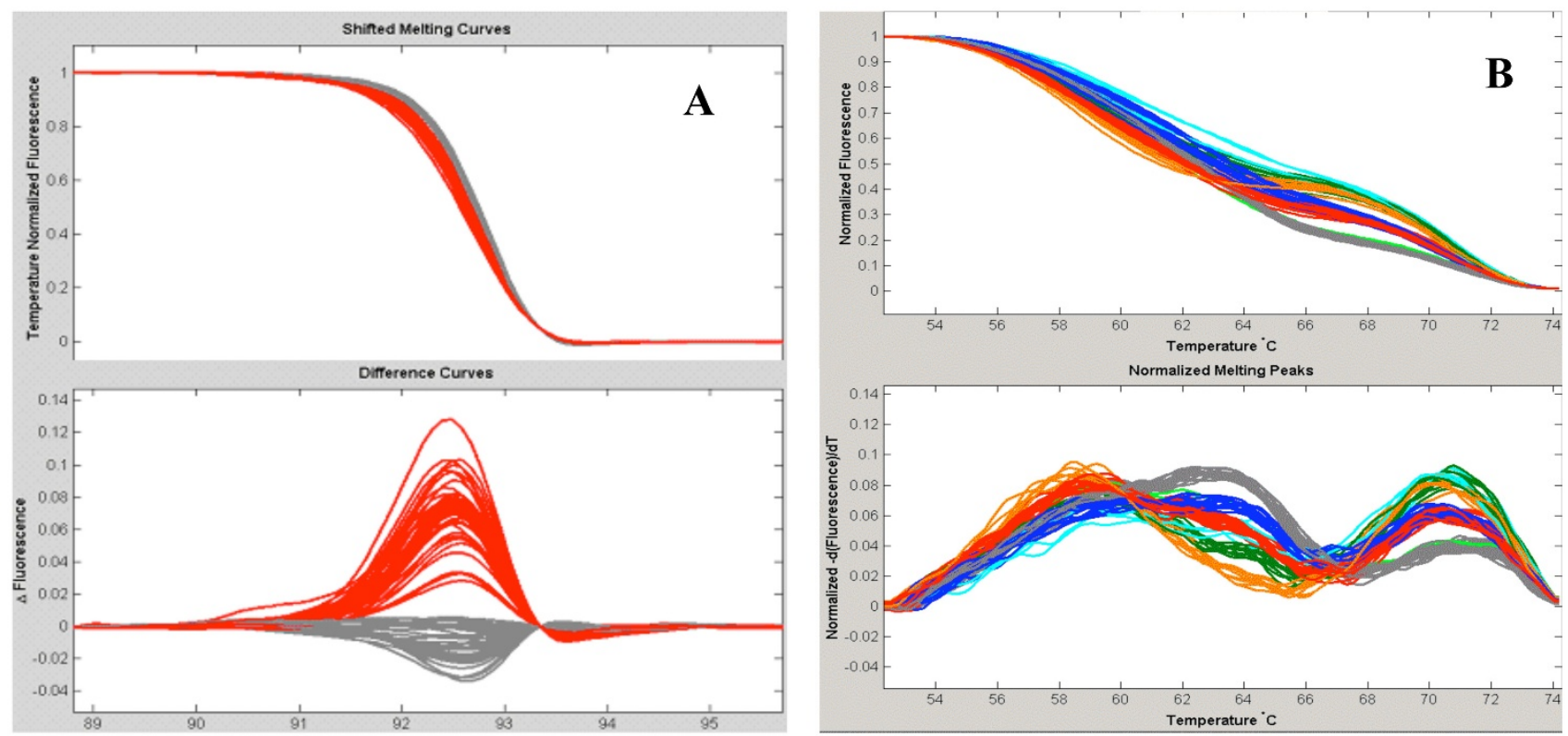

\begin{tabular}{|c|c|c|c|}
\hline & rs1641548 & rs1641549 & rs858528 \\
\hline grey & $\mathrm{G} / \mathrm{G}$ & $\mathrm{G} / \mathrm{G}$ & $\mathrm{G} / \mathrm{G}$ \\
\hline red & $\mathrm{G} / \mathrm{A}$ & $\mathrm{G} / \mathrm{G}$ & $\mathrm{G} / \mathrm{A}$ \\
\hline dark blue & $\mathrm{G} / \mathrm{G}$ & $\mathrm{G} / \mathrm{G}$ & $\mathrm{G} / \mathrm{A}$ \\
\hline dark green & $\mathrm{G} / \mathrm{A}$ & $\mathrm{G} / \mathrm{G}$ & $\mathrm{A} / \mathrm{A}$ \\
\hline orange & $\mathrm{A} / \mathrm{A}$ & $\mathrm{G} / \mathrm{G}$ & $\mathrm{A} / \mathrm{A}$ \\
\hline light blue & $\mathrm{G} / \mathrm{G}$ & $\mathrm{G} / \mathrm{G}$ & $\mathrm{A} / \mathrm{A}$ \\
\hline light green & $\mathrm{G} / \mathrm{A}$ & $\mathrm{G} / \mathrm{A}$ & $\mathrm{G} / \mathrm{A}$ \\
\hline
\end{tabular}

\section{Figure 5}

Simultaneous genotyping of rs858528, rs I 64 I 548, and rs I 64 I 549 using two unlabeled probes. A Samples that are heterozygous for rs858528, rs 1641548, and rs I64I549 have essentially indistinguishable melting curves (in red). B Genotyping using two unlabeled probes. Probe I targets the $G$ allele of rs858528 and probe 2 targets the $G$ allele of rs I64I548 and the A allele of rs I64I549. C Each distinct melting profile from panel B corresponds to a combination of genotypes of the three SNPs found in our population.

on partial or full coding sequence(s) of the genes of interest. For instance, more than $80 \%$ of TP53 mutation studies focus on exons 5-8 (residues 126-306) because most mutations are localized in the DNA binding domain of the protein (residues 100-300) [21,22]. However, in one study where the HRM analysis was extended to the entire coding exon of TP53, $41 \%$ of the alterations fall outside exons 5-8 [22]. Thus partial scanning of TP53 sequence may lead to a bias in the mutation analysis. Following this idea, we aimed to evaluate a set of HRM assays that sample the entire TP53 locus, including one or more amplicons from the proximal promoter, coding exons, introns, and 3' UTR. Our study thereby provides a broader view of the strengths and limitations of HRM-based techniques. However, the TP53 amplicons used in the present study were not designed specifically for HRM but rather for mutation screening of the entire TP53 locus by direct resequencing in the context of a Li-Fraumeni syndromerelated study (Garritano et al, in preparation). Consequently, some of the amplicons were longer than the optimum for HRM mutation scanning. Nonetheless, we have shown that the sensitivity of HRM for mutation screening is very high, especially for amplicons $<400 \mathrm{bp}$.

For genotyping applications, especially for intronic SNPs, primers were sometimes designed quite far from the SNP of interest to avoid unspecific amplification. Despite the length of the amplicons used, we obtained full concordances between HRM genotyping calls and results of direct sequencing. Moreover, using an amplicon of $>400 \mathrm{bp}$, we 

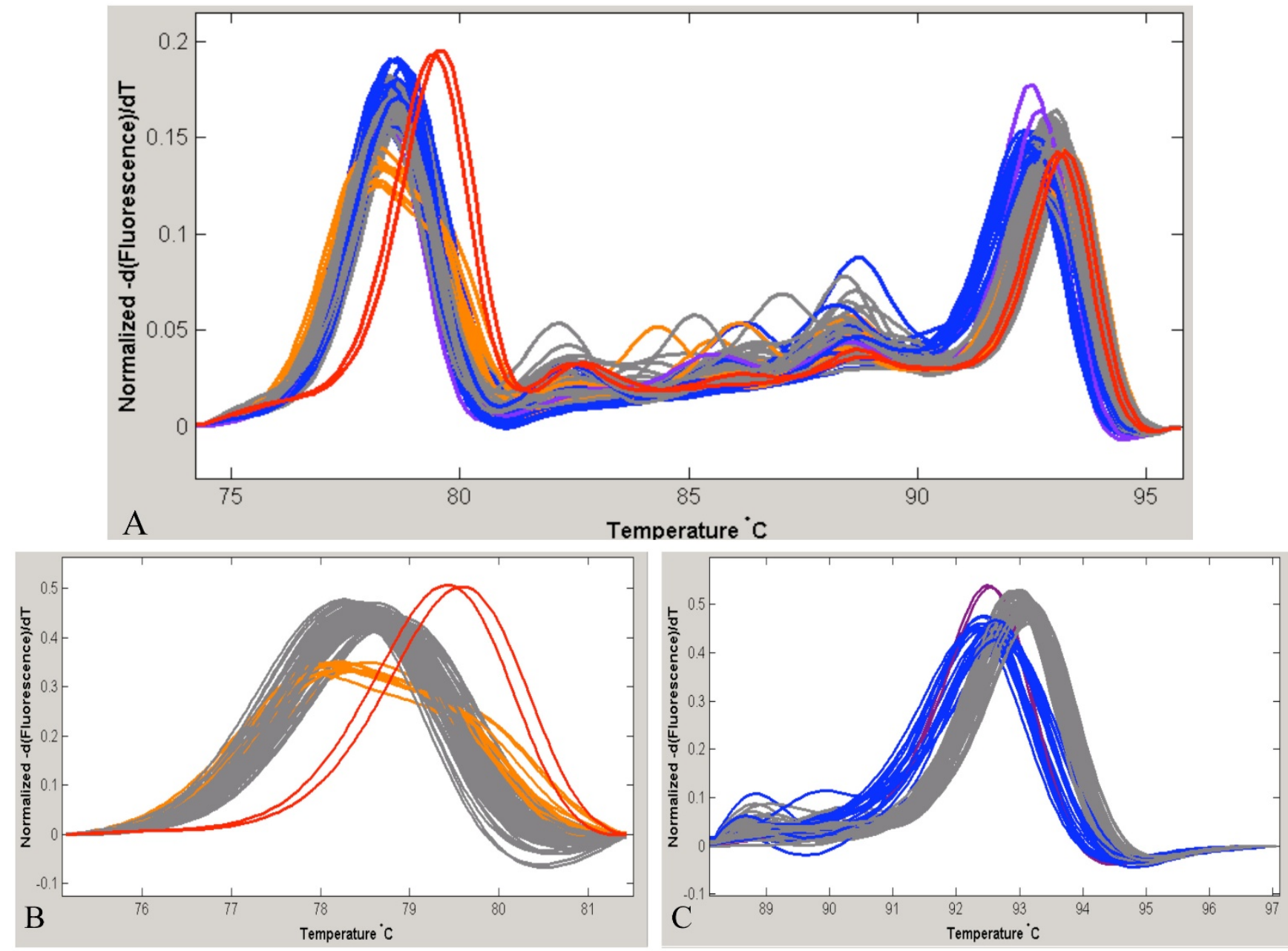

\section{Figure 6}

An amplicon containing rs9903378 and an amplicon containing rs9894946 were amplified in a multiplex PCR. A The melting curves of the different genotype combinations of the two SNPs show different profiles. In gray TT-CC, in blue TT-CT, in red GG-CC, in orange TG-CC and in purple TT-TT, respectively for rs 9903378 and rs 9894946 . B The analysis was performed in the region of melting temperature of rs $9903378\left(75-80^{\circ} \mathrm{C}\right)$. in gray TT, in orange TG in red GG. C The analysis was performed in the region of melting temperature of rs $9894946\left(90-95^{\circ} \mathrm{C}\right)$. in gray CC, in blue CT in purple TT.

succeeded to simultaneously genotype two SNPs located approximately 200 bp apart from each other (SNP rs858528 and rs1641549), thus reducing the number of PCR reactions and improving time and cost effectiveness.

In mutation scanning mode, HRM tended to call as "variant" some DNA samples that actually were wild type. To minimize the frequency of false positive "variant" calls, it is recommended to standardize DNA preparation, storage methods, and storage conditions. Because the sensitivity and specificity of HRM are exquisitely dependent on the melting temperature of each individual sample, variation in salt or buffer concentration carried into PCR reactions along with substrate DNA can generate heterogeneous melting profiles. If needed, to reduce sample-to-sample heterogeneity, it can also be useful to perform a nested PCR and the HRM assay on the secondary PCR.

In the course of our work, we have observed one potential weakness in HRM: the technique may have limited sensitivity for single nucleotide insertion-deletion variants located immediately adjacent to mononucleotide runs of sufficient length that they stutter during PCR. In this work, SNP rs17551157, an insertion of a cytosine adjacent to a 7 cytosine repeat within TP53 proximal promoter, was undetectable in a 653 bp HRM amplicon. A similar situation was also encountered during a large-scale case/control mutation scanning of ATM performed in our laboratory, where insertion of an adenosine adjacent to an intronic run of 10 thymines (rs3218681) was also unde- 
tectable by HRM mutation scanning. In both cases, the sequencing chromatograms revealed PCR stuttering at the mononucleotide run (data not shown). Such mononucleotide runs are relatively uncommon within the ORF of protein coding genes. Nonetheless, we suggest that at the outset of an HRM based mutation-screening project, investigators check the ORF of the gene of interest for mononucleotide runs that could create such a problem. If any are present, apply a different mutation screening technique to the relevant amplicon.

From our extensive mutation screening of the TP53 locus, we found that HRM provides sensitive assays both for detection of new sequence variants and genotyping of known polymorphisms. Table 2 summarizes the various HRM approaches for the different genetic contexts that we have considered, according to the number of SNPs present in each amplicon and to their frequencies in the studied population. In our experience, selecting an appropriate HRM analysis strategy depends both on study size and the number of known common polymorphisms in a given amplicon. For relatively small mutation screening studies, it may be reasonable to sequence all samples that appear to contain a sequence variant. In this case, amplicons known (or found) to contain a common variant can be PCR amplified in duplicate, once as a standard analysis and once as a spike-in analysis. The former will detect the presence of heterozygous variants and the latter will detect the presence of minor allele homozygotes. All samples with HRM curves that differ from the major allele homozygotes curves would then be queued for sequencing.

In large-scale mutation screening studies, there may be cost benefit to enabling HRM determination of common SNP genotypes prior to mutation scanning, so that only samples showing a variant HRM curve not attributable to the presence of a common SNP are queued for sequencing.
If an amplicon contains a common variant, this variant can mask the presence of a rare variant that might have the same melting profile. Without a discrimination step, one has to either 1) sequence all the heterozygous samples, even though most will be due to a common SNP or 2) accept failure to detect rare variants that have the same melting profile as the common SNP. Inclusion of a discrimination step, which can be achieved with little added cost and with no extra PCR reactions, allows assigning the common SNP by genotyping and simultaneously queuing the rare variant heterozygotes for identification by sequencing.

\section{Conclusion}

HRM is a simple and cost effective post-PCR technique that can be used for high-throughput mutation scanning and genotyping in a small laboratory environment. It is inexpensive, flexible, and only mildly constrained by primer design. HRM reactions are closed-tube, which reduces risk of contamination. In addition, HRM assays are non-destructive so that the actual sample used in mutation scanning can serve as a sequencing template.

\section{Abbreviations}

dHPLC: denaturated High-Performance Liquid Chromatography; HRM: High-Resolution Melting curve analysis; SNP: Single Nucleotide Polymorphisms.

\section{Authors' contributions}

SG carried out the experiments and drafted the manuscript; FG participated in its coordination and helped to draft the manuscript; CV developed the Laboratory Information Management Systems (LIMS); TND participated in design of the unlabeled 3' blocked probe assays for each common SNP; FLK participated in the development of the laboratory workflow; DD participated in the experiment design; FL participated in its coordination and helped to draft the manuscript; SL participated in its coordination and helped to draft the manuscript; SVT conceived the study, participated in its design and

Table 2: Summary of different approaches utilized for each genetic situations.

\begin{tabular}{ll}
\hline Genetic situation & HRM solutions \\
\hline No common SNP in the amplicon & Direct HRM analysis \\
\hline Only one common SNP in the amplicon & $\begin{array}{l}\text { Sometimes it is possible to distinguish directly the three groups (Figure I) } \\
\text { Otherwise, spike-in or unlabelled probe is needed (Figure 2) }\end{array}$ \\
\hline Two SNPs in the amplicon, one is common the other is rare & $\begin{array}{l}\text { Spike-in when possible (Figure 3); otherwise, use unlabeled probe for the common } \\
\text { SNP, and perform mutation scanning for the rare one (Figure 4) }\end{array}$ \\
\hline $\begin{array}{l}\text { Two or more common SNPs in the amplicon } \\
\text { Two SNPs in two different amplicons }\end{array}$ & $\begin{array}{l}\text { Use an unlabeled probe for each SNP (Figure 5) } \\
\text { When T } \text { of } 2 \text { amplicons is different, it is possible to perform multiplex PCR } \\
\text { (Figure 6) }\end{array}$
\end{tabular}


coordination and helped to draft the manuscript. All authors read and approved the final manuscript.

\section{Additional material}

\section{Additional file 1}

Primers sequences for TP53 SNPs analyzed in the present study. The data provided correspond to the oligonucleotide sequences used to analyze TP53 SNPs.

Click here for file

[http://www.biomedcentral.com/content/supplementary/14712156-10-5-S1.doc]

\section{Acknowledgements}

SG is a recipient of a fellowship from the University of Pisa and a Special Training Award from the International Agency for Research on Cancer. TN is a recipient of a fellowship from the Fondation de France. We would like to thank Dr Pierre Hainaut for providing breast cancer cell-lines and $\mathrm{Dr}$ Maria Isabel Waddington Achatz for supplying us with Li Fraumeni samples. We also thank Ms Nathalie Forey and Ms Sandrine McKay-Chopin for their technical assistance.

\section{References}

I. Nickerson DA, Rieder MJ, Crawford DC, Carlson CS, Livingston RJ: An overview of the environmental genome project. Essays on the Future of Environmental Health Research: A Tribute to Dr Keneth Olden 2005:42-53.

2. Guthery SL, Salisbury BA, Pungliya MS, Stephens JC, Bamshad M: The structure of common genetic variation in United States populations. Am J Hum Genet 2007, 81:|22|-1231.

3. Gorlov IP, Gorlova OY, Sunyaev SR, Spitz MR, Amos Cl: Shifting paradigm of association studies: value of rare single-nucleotide polymorphisms. Am J Hum Genet 2008, 82: I00-I I 2.

4. Lin HF, Juo $\mathrm{SH}$, Cheng R: Comparison of the power between microsatellite and single-nucleotide polymorphism markers for linkage and linkage disequilibrium mapping of an electrophysiological phenotype. BMC Genet 2005, 6(Suppl I):S7.

5. Papachristou C, Lin S: Microsatellites versus Single-Nucleotide Polymorphisms in confidence interval estimation of disease loci. Genet Epidemiol 2006, 30:3-17.

6. Gray IC, Campbell DA, Spurr NK: Single nucleotide polymorphisms as tools in human genetics. Hum Mol Genet 2000, 9:2403-2408.

7. Dong LM, Potter JD, White E, Ulrich CM, Cardon LR, Peters U: Genetic susceptibility to cancer: the role of polymorphisms in candidate genes. JAMA 2008, 299:2423-2436.

8. Lamba JK, Crews K, Pounds S, Schuetz EG, Gresham J, Gandhi V, Plunkett W, Rubnitz J, Ribeiro R: Pharmacogenetics of deoxycytidine kinase: identification and characterization of novel genetic variants. J Pharmacol Exp Ther 2007, 323:935-945.

9. Oldenburg J, Bevans CG, Fregin A, Geisen C, Muller-Reible C Watzka M: Current pharmacogenetic developments in ora anticoagulation therapy: the influence of variant VKORCI and CYP2C9 alleles. Thromb Haemost 2007, 98:570-578.

10. Zhu X, Yan D, Cooper RS, Luke A, Ikeda MA, Chang YP, Weder A, Chakravarti $A$ : Linkage disequilibrium and haplotype diversity in the genes of the renin-angiotensin system: findings from the family blood pressure program. Genome Res 2003 I3:173-181.

II. Yan D, Ouyang XM, Zhu X, Du LL, Chen ZY, Liu XZ: Refinement of the DFNA4I locus and candidate genes analysis. I Hum Genet 2005, 50:516-522.

12. Meaburn E, Butcher LM, Schalkwyk LC, Plomin R: Genotyping pooled DNA using 100 K SNP microarrays: a step towards genomewide association scans. Nucleic Acids Res 2006, 34:e27.

13. Rauch A, Rüschendorf F, Huang J, Trautmann U, Becker C, Thiel C Jones KW, Reis A, Nürnberg P: Molecular karyotyping using an
SNP array for genomewide genotyping. J Med Genet 2004 41:916-922.

14. Albert TJ, Molla MN, Muzny DM, Nazareth L, Wheeler D, Song X, Richmond TA, Middle CM, Rodesch MJ, Packard CJ, Weinstock GM, Gibbs RA: Direct selection of human genomic loci by microarray hybridization. Nat Methods 2007, 4:903-905.

15. Okou DT, Steinberg KM, Middle C, Cutler DJ, Albert TJ, Zwick ME Microarray-based genomic selection for high-throughput resequencing. Nat Methods 2007, 4:907-909.

16. Mariadason JM, Augenlicht LH, Arango D: Microarray analysis in the clinical management of cancer. Hematol Oncol Clin North Am 2003, 17:377-387.

17. Kashiwagi H, Uchida K: Genome-wide profiling of gene amplification and deletion in cancer. Hum Cell 2000, I3:|35-|4I.

18. Wade MM, Volokhov D, Peredelchuk M, Chizhikov V, Zhang Y: Accurate mapping of mutations of pyrazinamide-resistant Mycobacterium tuberculosis strains with a scanning-frame oligonucleotide microarray. Diagn Microbiol Infect Dis 2004, 49:89-97.

19. Skot L, Humphreys J, Humphreys MO, Thorogood D, Gallagher J, Sanderson R, Armstead IP, Thomas ID: Association of candidate genes with flowering time and water-soluble carbohydrate content in Lolium perenne (L.). Genetics 2007, I 77:535-547.

20. Tavtigian SV, Le Calvez-Kelm F: Molecular Diagnostics: Methods and Limitations. In Hereditary Breast Cancer Informa healthcare.Isaacs and Rebbeck.; 2008:I79-205.

21. Krypuy M, Ahmed AA, Etemadmoghadam D, Hyland SJ, Australian Ovarian Cancer Study Group, DeFazio A, Fox SB, Brenton JD, Bowtell DD, Dobrovic A: High resolution melting for mutation scanning of TP53 exons 5-8. BMC Cancer 2007, 7:168.

22. Bastien R, Lewis TB, Hawkes JE, Quackenbush JF, Robbins TC, Palazzo J, Perou CM, Bernard PS: High-throughput amplicon scanning of the TP53 gene in breast cancer using high-resolution fluorescent melting curve analyses and automatic mutation calling. Hum Mutat 2008, 29:757-764.

23. Millat G, Chanavat V, Rodriguez-Lafrasse C, Rousson R: Rapid, sensitive and inexpensive detection of SCN5A genetic variations by high resolution melting analysis. Clin Biochem 2008.

24. Takano EA, Mitchell G, Fox SB, Dobrovic A: Rapid detection of carriers with BRCAI and BRCA2 mutations using high resolution melting analysis. BMC Cancer 2008, 8:59.

25. Audrezet MP, Dabricot A, Le MC, Ferec C: Validation of high-resolution DNA melting analysis for mutation scanning of the cystic fibrosis transmembrane conductance regulator (CFTR) gene. I Mol Diagn 2008, I 0:424-434.

26. Laurie AD, George PM: Evaluation of high-resolution melting analysis for screening the LDL receptor gene. Clin Biochem 2008.

27. Sarvary E, Nagy P, Benjamin A, Szoke M, Remport A, Jansen J, Nemes B, Kobori L, Fehervari I, Sulyok B, Perner F, Varga M, Fazakas J, Lakatos $M$, Szabo $M$, Toth $A$, Járay J: Mutation scanning of the p53 tumor suppressor gene in renal and liver transplant patients in Hungary. Transplant Proc 2005, 37:969-972.

28. Voegele C, Tavtigian SV, de Silva D, Cuber S, Thomas A, Le CalvezKelm F: A Laboratory Information Management System (LIMS) for a high throughput genetic platform aimed at candidate gene mutation screening. Bioinformatics 2007, 23:2504-2506.

29. Zhou L, Myers AN, Vandersteen JG, Wang L, Wittwer CT: Closedtube genotyping with unlabeled oligonucleotide probes and a saturating DNA dye. Clin Chem 2004, 50:I328-I335.

30. Zhou L, Wang L, Palais R, Pryor R, Wittwer CT: High-resolution DNA melting analysis for simultaneous mutation scanning and genotyping in solution. Clin Chem 2005, 51:1770-1777.

31. Montgomery J, Wittwer CT, Palais R, Zhou L: Simultaneous mutation scanning and genotyping by high-resolution DNA melting analysis. Nat Protoc 2007, 2:59-66. 